\title{
Convergence of Variational Iteration Method for Solving Singular Partial Differential Equations of Fractional Order
}

\author{
Asma Ali Elbeleze, ${ }^{1}$ Adem Kılıçman, ${ }^{2}$ and Bachok M. Taib ${ }^{1}$ \\ ${ }^{1}$ Faculty of Science and Technology, Universiti Sains Islam Malaysia, 71800 Nilai, Malaysia \\ ${ }^{2}$ Department of Mathematics, Faculty of Science, University Putra Malaysia, 4300 Serdang, Selangor, Malaysia \\ Correspondence should be addressed to Adem Kılıçman; akilic@upm.edu.my
}

Received 5 March 2014; Revised 16 May 2014; Accepted 10 June 2014; Published 16 July 2014

Academic Editor: Dumitru Baleanu

Copyright ( 2014 Asma Ali Elbeleze et al. This is an open access article distributed under the Creative Commons Attribution License, which permits unrestricted use, distribution, and reproduction in any medium, provided the original work is properly cited.

We are concerned here with singular partial differential equations of fractional order (FSPDEs). The variational iteration method (VIM) is applied to obtain approximate solutions of this type of equations. Convergence analysis of the VIM is discussed. This analysis is used to estimate the maximum absolute truncated error of the series solution. A comparison between the results of VIM solutions and exact solution is given. The fractional derivatives are described in Caputo sense.

\section{Introduction}

In recent years, considerable attention has been devoted to the study of the fractional calculus and its numerous applications in many areas such as physics and engineering. The applications of fractional calculus used in many fields such as electrical networks, control theory of dynamical systems, probability and statistics, electrochemistry of corrosion, chemical physics, optics, and signal processing can be successfully modeled by linear or nonlinear FDEs [1-7]. Further, fractional partial differential equations appeared in many fields of engineering and science, including fractals theory, statistics, fluid flow, control theory, biology, chemistry, diffusion, probability, and potential theory $[8,9]$.

The singular partial differential equations of fractional order (FSPDEs), as generalizations of classical singular partial differential equations of integer order (SPDEs), are increasingly used to model problems in physics and engineering. Consequently, considerable attention has been given to the solution of singular partial differential equations of fractional order. Finding approximate or exact solutions of SPDEs is an important task. Except for a limited number of these equations, we have difficulty in finding their analytical solutions. Therefore, there have been attempts to find methods for obtaining approximate solutions. Several such techniques have drawn special attention, such as variational iteration method [10], homotopy analysis method [11], and homotopy iteration method [12].

The variational iteration method (VIM) was proposed by $\mathrm{He}$ [13-16] due to its flexibility and convergence and efficiently works with different types of linear and nonlinear partial differential equations of fractional order and gives approximate analytical solution for all these types of equations without linearization or discretization; many author have been studying it; for example, see [17-21]. In this paper, we discuss the VIM for solving FSPDEs and obtain the convergence results of this method. The contribution of this work can be summarized in three points.

(1) Based on the sufficient condition that guarantees the existence of a unique solution to our problem (see Theorem 6) and using the series solution, convergence of VIM is discussed (see Theorem 7).

(2) Using point one, the maximum absolute truncated error of series solution of VIM is estimated (see Theorem 8).

(3) Some numerical examples are given. 
Consider fractional singular partial differential equations with variable coefficients

$$
\begin{array}{r}
\frac{\partial^{\alpha} u}{\partial t^{\alpha}}+\mu(x) \frac{\partial^{4} u}{\partial x^{4}}+\lambda(y) \frac{\partial^{4} u}{\partial y^{4}}+h(z) \frac{\partial^{4} u}{\partial z^{4}}=0, \\
a<x, y, z<b, t>0
\end{array}
$$

where the variable coefficients subject to initial conditions

$$
\begin{aligned}
u(x, y, z, 0) & =f_{0}(x, y, z), \\
\frac{\partial u}{\partial t}(x, y, z, 0) & =f_{1}(x, y, z)
\end{aligned}
$$

and boundary conditions

$$
\begin{aligned}
u(a, y, z, t)= & g_{0}(y, z, t), \quad u(b, y, z, t)=g_{1}(y, z, t) \\
u(x, a, z, t)= & g_{2}(x, z, t), \quad u(x, b, z, t)=g_{3}(x, z, t) \\
u(x, y, a, t)= & g_{4}(x, z, t), \quad u(x, y, b, t)=g_{5}(x, z, t) \\
& \frac{\partial^{2} u}{\partial x^{2}}(a, y, z, t)=k_{0}(y, z, t), \\
& \frac{\partial^{2} u}{\partial x^{2}}(b, y, z, t)=k_{0}(y, z, t), \\
& \frac{\partial^{2} u}{\partial y^{2}}(x, a, z, t)=k_{2}(x, z, t), \\
& \frac{\partial^{2} u}{\partial y^{2}}(x, a, z, t)=k_{3}(x, z, t), \\
& \frac{\partial^{2} u}{\partial z^{2}}(x, y, a, t)=k_{4}(x, y, t), \\
& \frac{\partial^{2} u}{\partial z^{2}}(x, y, b, t)=k_{5}(y, z, t),
\end{aligned}
$$

where $\partial^{\alpha} / \partial t^{\alpha}$ is the fractional derivative in the Caputo sense, $a<x, y, z<b$, and $g_{i}$ and $k_{i}, i=0, \ldots, 5$ are continuous. The $\partial^{4} u / \partial x^{4}, \partial^{4} u / \partial y^{4}$, and $\partial^{4} u / \partial z^{4}$ are linear bounded operator; that is, it is possible to find numbers $m_{1}, m_{2}, m_{3}>0$ such that $\left\|\partial^{4} u / \partial x^{4}\right\| \leq m_{1}\|u\|,\left\|\partial^{4} u / \partial y^{4}\right\| \leq m_{2}\|u\|,\left\|\partial^{4} u / \partial z^{4}\right\| \leq$ $m_{3}\|u\|$. Equation (1) can be written as

$$
\begin{array}{r}
{ }^{c} D_{t}^{\alpha} u(x, y, z, t) \\
=f\left(t, u(x, y, z, t), D_{x}^{n_{1}} u(x, y, z, t),\right. \\
\left.\quad D_{y}^{n_{2}} u(x, y, z, t), D_{z}^{n_{3}} u(x, y, z, t)\right),
\end{array}
$$

where $n_{1}=n_{2}=n_{3}=4$.

\section{Preliminaries}

In this section, we give some basic definitions and properties of fractional calculus theory used in this paper.
Definition 1. A real function $f(x), x>0$ is said to be in space $C \mu, \mu \in R$ if there exists a real number $p>\mu$, such that $f(x)=x^{p} f_{1}(x)$, where $f_{1}(x) \in C(0, \infty)$, and it is said to be in the space $C_{\mu}^{n}$ if $f^{n} \in R_{\mu}, n \in N$.

Definition 2. The Riemann-Liouville fractional integral operator of order $\alpha \geq 0$ of a function $f \in C \mu, \mu \geq-1$ is defined as

$$
\begin{array}{r}
J^{\alpha} f(x)=\frac{1}{\Gamma(\alpha)} \int_{0}^{x}(x-t)^{\alpha-1} f(t) d t \\
\alpha>0, t>0 .
\end{array}
$$

In particular $J^{0} f(x)=f(x)$.

For $\beta \geq 0$ and $\gamma \geq-1$, some properties of the operator $J^{\alpha}$ are

(1) $J^{\alpha} J^{\beta} f(x)=J^{\alpha+\beta} f(x)$,

(2) $J^{\alpha} J^{\beta} f(x)=J^{\beta} J^{\alpha} f(x)$,

(3) $J^{\alpha} x^{\gamma}=(\Gamma(\gamma+1) / \Gamma(\alpha+\gamma+1)) x^{\alpha+\gamma}$.

Definition 3. The Caputo fractional derivative of $f \in C_{-1}^{m}$, $m \in N$ is defined as

$$
\begin{array}{r}
D^{\alpha} f(x)=\frac{1}{\Gamma(m-\alpha)} \int_{0}^{x}(x-t)^{m-\alpha-1} f^{m}(t) d t, \\
m-1<\alpha \leq m .
\end{array}
$$

Lemma 4. If $m-1<\alpha \leq m, m \in N, f \in C_{\mu}^{m}, \mu>-1$, then the following two properties hold:

(1) $D^{\alpha}\left[J^{\alpha} f(x)\right]=f(x)$,

(2) $J^{\alpha}\left[D^{\alpha} f(x)\right]=f(x)-\sum_{k=1}^{m-1} f^{k}(0)\left(x^{k} / k !\right)$.

Lemma 5. Suppose that $u$ and their partial derivatives are continuous; then the fractional derivative, ${ }^{c} D_{t}^{\alpha} u(x, y, z, t)$, is bonded.

Proof. We need to prove that it is possible to find number $M>$ 0 such that $\left\|^{c} D_{t}^{\alpha} u(x, y, z, t)\right\| \leq M\|u\|$. From the definition of Caputo fractional derivative above we have

$$
\begin{aligned}
& \left\|{ }^{c} D_{t}^{\alpha} u(x, y, z, t)\right\| \\
& \quad=\left\|\frac{1}{\Gamma(m-\alpha)} \int_{a}^{b}(x-t)^{m-\alpha-1} u^{(m)}(t) d t\right\| \\
& \quad \leq \frac{|b-a|}{|(m-\alpha) \Gamma(m-\alpha)|}\|u\|=M\|u\|,
\end{aligned}
$$

where $M=|b-a| /|(m-\alpha) \Gamma(m-\alpha)|$.

\section{Analysis of the Variational Iteration Method}

To solve the fractional singular partial differential equations (4) by using the variational iteration method, with initial and 
boundary conditions (2) and (3), where $\left\|^{c} D_{t}^{\alpha} u(t)\right\|=M\|u\|$, we construct the following correction functional:

$$
\begin{aligned}
& u_{n+1}(x, y, z, t) \\
& =u_{n}(x, y, z, t) \\
& +J_{t}^{\alpha}\left[{ }^{c} D_{t}^{\alpha} u(x, y, z, t)\right. \\
& -f\left((x, y, z, t), u_{n}(x, y, z, t),\right. \\
& D_{x}^{n_{1}} u(x, y, z, t), D_{y}^{n_{2}} u_{n}(x, y, z, t), \\
& \left.\left.D_{z}^{n_{3}} u_{n}(x, y, z, t)\right)\right]
\end{aligned}
$$

or

$$
\begin{aligned}
& u_{n+1}(x, y, z, t) \\
& =u_{n}(x, y, z, t)+\frac{1}{\Gamma(\alpha)} \\
& \quad \times \int_{0}^{t}(t-s)^{\alpha-1} \lambda(s) \\
& \quad \times\left({ }^{c} D_{t}^{\alpha} u(x, y, z, s)\right. \\
& \quad-f\left(s, u_{n}(x, y, z, s), D_{x}^{n_{1}} u_{n}(x, y, z, s),\right. \\
& \left.\left.\quad D_{y}^{n_{2}} u_{n}(x, y, z, s), D_{z}^{n_{3}} u_{n}(x, y, z, s)\right)\right) d s .
\end{aligned}
$$

$J_{t}^{\alpha}$ is the Riemann-Liouville fractional integral operator of order $\alpha$, with respect to variable $t$, and $\lambda$ is a general Lagrange multiplier which can be identified as optimally variational theory [22], and $\widetilde{u}_{n}(x, t)$ are considered as restricted variation; that is, $\delta \widetilde{u}_{n}(x, t)=0$.

Making the above correction functional stationary, the following condition can be obtained:

$$
\begin{aligned}
& \delta u_{k+1}(x, y, z, t) \\
& =\delta u_{n}(x, y, z, t)+\frac{1}{\Gamma(\alpha)} \delta \\
& \quad \times \int_{0}^{t}(t-s)^{\alpha-1} \lambda(s) \\
& \quad \times\left({ }^{c} D_{t}^{\alpha} u(x, y, z, s)\right. \\
& -f\left(t, \widetilde{u}_{n}(x, y, z, s), D_{x}^{n_{1}} \widetilde{u}_{n}(x, y, z, s),\right. \\
& \left.\left.D_{y}^{n_{2}} \widetilde{u}_{n}(x, y, z, s), D_{z}^{n_{3}} \widetilde{u}_{n}(x, y, z, s)\right)\right) d s
\end{aligned}
$$

and yields to Lagrange multiplier

$$
\lambda(s)=s-t .
$$

We obtain the following iteration formula by substitution of (11) in (9)

$$
\begin{aligned}
& u_{n+1}(x, y, z, t) \\
& =u_{n}(x, y, z, t)+\frac{1}{\Gamma(\alpha-1)} \\
& \quad \times \int_{0}^{t}(t-s)^{\alpha-2}(t-s) \\
& \quad \times\left({ }^{c} D_{t}^{\alpha} u(x, y, z, s)\right. \\
& -f\left(s, u_{k}(x, y, z, s), D_{x}^{n_{1}} u_{n}(x, y, z, s),\right. \\
& \left.\left.\quad D_{y}^{n_{2}} u_{n}(x, y, z, s), D_{z}^{n_{3}} u_{n}(t)\right)\right) d s .
\end{aligned}
$$

That is,

$$
\begin{aligned}
& u_{n+1}(x, y, z, t) \\
& =u_{n}(x, y, z, t)-\frac{(\alpha-1)}{\Gamma(\alpha)} \\
& \quad \times \int_{0}^{t}(t-s)^{\alpha-1} \\
& \quad \times\left({ }^{c} D_{t}^{\alpha} u(x, y, z, s)\right. \\
& \quad-f\left(s, u_{n}(x, y, z, s),\right. \\
& \quad D_{x}^{n_{1}} u_{n}(x, y, z, s), \\
& D_{y}^{n_{2}} u_{n}(x, y, z, s), \\
& \left.\left.\quad D_{z}^{n_{3}} u_{n}(x, y, z, s)\right)\right) d s .
\end{aligned}
$$

This yields the following iteration formula:

$$
\begin{aligned}
& u_{n+1}(x, y, z, t) \\
& =u_{n}(x, y, z, t)-(\alpha-1) \\
& \times J_{t}^{\alpha}\left[{ }^{c} D_{t}^{\alpha} u(x, y, z, t)\right. \\
& -f\left(t, u_{n}(x, y, z, t),\right. \\
& D_{x}^{n_{1}} u(x, y, z, t), D_{y}^{n_{2}} u_{n}(x, y, z, t), \\
& \left.\left.D_{z}^{n_{3}} u_{n}(t)\right)\right] .
\end{aligned}
$$

The initial approximation $u_{0}$ can be chosen by the following manner which satisfies initial conditions:

$$
u_{0}=\sum_{j=0}^{1} \gamma_{j} \frac{t^{j}}{j !}=\gamma_{0}+\gamma_{1} t
$$

where $\gamma_{0}=f_{0}(x, y, z), \gamma_{1}=f_{1}(x, y, z)$. 
We can obtain the following first-order approximation by substitution of (15) into (14)

$$
\begin{aligned}
& u_{1}(x, y, z, t) \\
& =u_{0}(x, y, z, t)-(\alpha-1) J_{t}^{\alpha} \\
& \times\left[{ }^{c} D_{t}^{\alpha} u_{0}(x, y, z, t)\right. \\
& -f\left(t, u_{0}(t),\right. \\
& D_{x}^{n_{1}} u(x, y, z, t), D_{y}^{n_{2}} u_{0}(x, y, z, t), \\
& \left.\left.D_{z}^{3_{m}} u_{0}(x, y, z, t)\right)\right] .
\end{aligned}
$$

Finally, by substituting the constant values of $\gamma_{0}$ and $\gamma_{1}$ into (16), we have the results as the first approximate solutions of (4) with (2) and (3).

\subsection{Convergence Analysis}

3.1.1. Existence and Uniqueness Theorem. Define $F: X \rightarrow X$ contentious mapping, and the function $F\left(t, u_{0}, u_{1}, \ldots, u_{n-1}\right)$ exists with continuous and bounded derivatives, where $X$ is the Banach space $(C(J),\|\cdot\|)$, the space of all continuous functions on $J$ with the norm

$$
\|u\|=\max _{\forall t \in J}|u|
$$

and satisfies Lipschitz condition with Lipschitz constant $L$, such that

$$
\begin{gathered}
\mid f\left(t, u_{1}(x, y, z, t), D^{n_{1}} u_{1}(x, y, z, t),\right. \\
\left.D^{n_{2}} u_{1}(x, y, z, t), D^{n_{3}} u_{1}(x, y, z, t)\right) \\
-f\left(t, u_{2}(x, y, z, t), D^{n_{1}} u_{2}(x, y, z, t),\right. \\
\left.D^{n_{2}} u_{2}(x, y, z, t), D^{n_{m}} u_{2}(x, y, z, t)\right) \mid \\
\leq L \mid\left(u_{1}(x, y, z, t), D^{n_{1}} u_{1}(x, y, z, t),\right. \\
\left.\quad D^{n_{2}} u_{1} x, y, z,(t), D^{n_{3}} u_{1}(x, y, z, t)\right) \\
-\left(u_{2}(t), D^{n_{1}} u_{2}(x, y, z, t),\right. \\
\left.D^{n_{2}} u_{2}(x, y, z, t), D^{n_{3}} u_{2}(x, y, z, t)\right) \mid \\
\quad 0<L<1, t \geq 0 .
\end{gathered}
$$

Theorem 6. Let $f$ satisfy the Lipschitz condition (18) then the problem (4) with (2) and (3) has unique solution $u(x, t)$, whenever $0<L<1$.

Proof. (1) The existence of the solution. From equation (4) we have

$$
u=f\left(t, \sum_{j=0}^{m-1} c_{j} \frac{t^{j}}{j !}+J^{\alpha} u, J^{\alpha} D_{x}^{n_{1}} u, J^{\alpha} D_{y}^{n_{2}} u, J^{\alpha} D_{z}^{n_{3}} u\right)
$$

The mapping $F: X \rightarrow X$ is defined as

$$
F(u)=f\left(t, \sum_{j=0}^{m-1} c_{j} \frac{t^{j}}{j !}+J^{\alpha} u, J^{\alpha} D_{x}^{n_{1}} u, J^{\alpha} D_{y}^{n_{2}} u, J^{\alpha} D_{z}^{n_{3}} u\right) .
$$

Let $u, v \in X$; then

$$
\begin{aligned}
& |F(u)-F(v)| \\
& =\mid f\left(t, \sum_{j=0}^{m-1} c_{j} \frac{t^{j}}{j !}+J^{\alpha} u, J^{\alpha} D_{x}^{n_{1}} u, J^{\alpha} D_{y}^{n_{2}} u, J^{\alpha} D_{z}^{n_{3}} u\right) \\
& -f\left(t, \sum_{j=0}^{m-1} c_{j} \frac{t^{j}}{j !}+J^{\alpha} v, J^{\alpha} D_{x}^{n_{1}} v, J^{\alpha} D_{y}^{n_{2}} v, J^{\alpha} D_{z}^{n_{3}} v\right) \\
& \leq L \sum_{i=0}^{3}\left|J^{\alpha-n_{i}} u-J^{\alpha-n_{i}} v\right| \\
& \leq L \sum_{i=0}^{3}\left|\frac{1}{\Gamma\left(\alpha-n_{i}\right)} \int_{0}^{t}(t-s)^{\alpha-n_{i}-1}[u-v] d s\right| \\
& \max |F(u)-F(v)| \\
& \leq L \sum_{i=0}^{3} \frac{1}{\Gamma\left(\alpha-n_{i}\right)} \max \left|\int_{0}^{t}(t-s)^{\alpha-n_{i}-1}[u-v] d s\right| \\
& \leq L \sum_{i=0}^{3} \frac{\|u-v\|}{\Gamma\left(\alpha-n_{i}\right)}\left|\int_{0}^{t}(t-s)^{\alpha-n_{i}-1} d s\right| \\
& \leq \sum_{i=0}^{3} \frac{\mathrm{LMT}}{\Gamma\left(\alpha-n_{i}\right)}\|u-v\| \\
& \leq \gamma\|u-v\|,
\end{aligned}
$$

where $\gamma=\sum_{i=0}^{3}\left(\operatorname{LMT} /\left(\Gamma\left(\alpha-n_{i}\right)\right)\right)<1$, then we get

$$
\|F(u)-F(v)\| \leq\|u-v\|,
$$

therefore the mapping $F$ is contraction, and there exists unique solution $u \in C(J)$ to problem (4).

(2) The uniqueness of the solution (see [23]).

\subsubsection{Proof of Convergence}

Theorem 7. Suppose that $X$ is Banach space and $F: X \rightarrow X$ satisfies condition (18). Then, the sequence (14) converges to the solution of (4) with (2) and (3).

Proof. Defined $(C(J),\|\cdot\|)$ is the Banach space, the space of all continuous functions on $J$ with the norm

$$
\|u(x, y, z, t)\|=\max _{\forall t \in J}|u(x, y, z, t)| .
$$


We need to show that $\left\{u_{n}\right\}$ is a Cauchy sequence in this Banach space:

$$
\begin{aligned}
& \left\|u_{n}-u_{m}\right\| \\
& =\max \left|u_{n}-u_{m}\right| \\
& =\max \mid u_{n-1}-\frac{(\alpha-1)}{\Gamma(\alpha)} \\
& \times \int_{0}^{t}(t-s)^{\alpha-1} \\
& \times\left[D^{\alpha} u_{n-1}(x, y, z, s)\right. \\
& -F\left(s, u_{n-1}(s), D_{x}^{n_{1}} u_{n-1}(s),\right. \\
& \left.\left.D_{y}^{n_{2}} u_{n-1}(s), D_{z}^{n_{3}} u_{n-1}(s)\right)\right] d s \\
& -u_{m-1}+\frac{(\alpha-1)}{\Gamma(\alpha)} \\
& \times \int_{0}^{t}(t-s)^{\alpha-1} \\
& \times\left[D^{\alpha} u_{m-1}\right. \\
& -F\left(s, u_{m-1}(x, y, z, s) D_{x}^{n_{1}} u_{m-1},\right. \\
& \left.\left.D_{y}^{n_{2}} u_{m-1}, D_{z}^{n_{3}} u_{m-1}\right)\right] d s \\
& \leq \max \left[\left|u_{n-1}-u_{m-1}\right|\right. \\
& -\mid \frac{(\alpha-1)}{\Gamma(\alpha)} \\
& \times \int_{0}^{t}(t-s)^{\alpha-1}\left[D^{\alpha} u_{n-1}-D^{\alpha} u_{m-1}\right] \\
& \text { - F (s, un-1 } \left., D_{x}^{n_{1}} u_{n-1}, D_{y}^{n_{2}} u_{n-1}, D_{z}^{n_{3}} u_{n-1}\right) d s \\
& \left.-F\left(s, u_{m-1}, D_{x}^{n_{1}} u_{m-1}, D_{y}^{n_{2}} u_{m-1}, D_{z}^{n_{3}} u_{n-1}\right) d s \mid\right] \\
& \leq \max \left[\left|u_{n-1}-u_{m-1}\right|\right. \\
& -\frac{\left(M+\left(m_{1}+m_{2}+m_{3}\right) R T\right)}{\Gamma(\alpha)} \\
& \left.\times \int_{0}^{t}\left|(t-s)^{\alpha-1}\right|\left|u_{n-1}-u_{m-1}\right| d s\right] \\
& \leq \max \left|u_{n-1}-u_{m-1}\right| \\
& \begin{array}{c}
\times\left(1-\frac{\left(M+\left(m_{1}+m_{2}+m_{3}\right) R T\right)}{\Gamma(\alpha-1)}\right. \\
\left.\times \int_{0}^{t}\left|(t-s)^{\alpha-1}\right| d s\right),
\end{array}
\end{aligned}
$$

where

$$
R=\max \left|(t-s)^{\alpha-1}\right| .
$$

Finally, we have

$$
\begin{aligned}
& \left\|u_{n}-u_{m}\right\| \\
& \leq\left(1-\frac{\left(M+\left(m_{1}+m_{2}+m_{3}\right) R T\right)}{\Gamma(\alpha-1)}\right) \\
& \quad \times\left\|u_{n-1}-u_{m-1}\right\| \\
& \leq \gamma\left\|u_{n-1}-u_{m-1}\right\|,
\end{aligned}
$$

where $M, R, T, \Gamma(\alpha)$ are constants and

$$
\gamma=\left(1-\frac{\left(M+\left(m_{1}+m_{2}+m_{3}\right) R T\right)}{\Gamma(\alpha-1)}\right) .
$$

Let $n=m+1$. Then

$$
\begin{aligned}
& \left\|u_{m+1}-u_{m}\right\| \\
& \quad \leq \gamma\left\|u_{m}-u_{m-1}\right\| \leq \gamma^{2}\left\|u_{m-1}-u_{m-2}\right\| \\
& \quad \leq \cdots \leq \gamma^{m}\left\|u_{1}-u_{0}\right\| .
\end{aligned}
$$

From the triangle inequality, we have

$$
\begin{aligned}
\left\|u_{n}-u_{m}\right\| & \\
\leq & \left\|u_{m+1}-u_{m}\right\|+\left\|u_{m+2}-u_{m+1}\right\| \\
\leq & \cdots \leq\left\|u_{n}-u_{n-1}\right\| \\
\leq & \gamma^{m}\left\|u_{1}-u_{0}\right\|+\gamma^{m+1}\left\|u_{1}-u_{0}\right\| \\
& +\cdots+\gamma^{n-1}\left\|u_{1}-u_{0}\right\| \\
\leq & {\left[\gamma^{m}+\gamma^{m+1}+\gamma^{m+2}+\cdots+\gamma^{n-1}\right]\left\|u_{1}-u_{0}\right\| } \\
\leq & \gamma^{m}\left[1+\gamma+\gamma^{2}+\cdots+\gamma^{n-m-1}\right]\left\|u_{1}-u_{0}\right\| \\
\leq & \gamma^{m}\left(\frac{1-\gamma^{n-m}}{1-\gamma}\right)\left\|u_{1}-u_{0}\right\| .
\end{aligned}
$$

Since $0<\gamma<1$, so $1-\gamma^{n-m}<1$, and then

$$
\left\|u_{n}-u_{m}\right\| \leq \frac{\gamma^{m}}{1-\gamma}\left\|u_{1}-u_{0}\right\| .
$$

But $\left\|u_{1}-u_{0}\right\|<\infty$; then $\left\|u_{n}-u_{m}\right\| \rightarrow 0$ as $m \rightarrow \infty$. We conclude that $u_{n}$ is a Cauchy sequence in $C[J]$, so the sequence converges and the proof is complete.

\subsubsection{Error Analysis}

Theorem 8. The maximum absolute error of the approximate solution $u_{m}$ to problem (4)-(3) is estimated to be

$$
\max _{t \in J}\left|u_{\text {exact }}-u_{n}\right| \leq k
$$


where

$$
\begin{aligned}
& k=\left(\frac{\gamma^{m}\left(M+\left(m_{1}+m_{2}+m_{3} R T\right) \beta\right)}{(1-\gamma)}\right)\left\|u_{0}\right\|, \\
& \beta=\left|\left(\frac{\alpha-1}{\Gamma(\alpha)}\right)\right| .
\end{aligned}
$$

Proof. From Theorem (9) and inequality (30) we have

$$
\left\|u_{n}-u_{m}\right\| \leq\left(\frac{\gamma^{m}}{1-\gamma}\right)\left\|u_{1}-u_{0}\right\|
$$

as $n \rightarrow \infty$; then $u_{n} \rightarrow u_{\text {exact }}$ and

$$
\begin{aligned}
\left\|u_{1}-u_{0}\right\| & \\
=\max _{t \in J} \mid & -\frac{(\alpha-1)}{\Gamma(\alpha)} \int_{0}^{t}(t-s)^{\alpha-1} \\
\times & {\left[D^{\alpha} u_{0}\right.} \\
& \left.-F\left(s, u_{0}, D_{x}^{n_{1}} u_{0}, D_{y}^{n_{2}}, D_{z}^{n_{3}} u_{0}\right)\right] d s \mid \\
=[(M+ & \left.\left.\left(m_{1}+m_{2}+m_{3} R T\right) \beta\right)\right]\left\|u_{0}\right\|,
\end{aligned}
$$

where $\beta=|((\alpha-1) / \Gamma(\alpha))|$, and thus, the maximum absolute error in the interval $J$ is

$$
\left\|u_{\text {exact }}-u_{n}\right\| \leq \max _{t \in J}\left|u_{\text {exact }}-u_{n}\right| \leq k .
$$

This completes the proof.

\section{Numerical Examples}

Example 1. Consider the following fourth-order fractional singular partial differential equation:

$$
\begin{array}{r}
\frac{\partial^{\alpha} u}{\partial t^{\alpha}}+\left(\frac{1}{x}+\frac{x^{4}}{120}\right) \frac{\partial^{4} u}{\partial x^{4}}=0, \\
\frac{1}{2}<x<1, \quad t>0, \quad 1<\alpha \leq 2 .
\end{array}
$$

With initial conditions

$$
\begin{aligned}
u(x, 0) & =0, \quad \frac{1}{2}<x<1, \\
\frac{\partial u}{\partial t}(x, 0) & =1+\frac{x^{5}}{120}, \quad 0<x<1
\end{aligned}
$$

and boundary conditions

$$
\begin{aligned}
u\left(\frac{1}{2}, t\right) & =\left(1+\frac{(1 / 2)^{5}}{120}\right) \sin t \\
u(1, t) & =\frac{121}{120} \sin t, \quad t>0, \\
\frac{\partial^{2} u}{\partial x^{2}}\left(\frac{1}{2}, t\right) & =\frac{1}{6}\left(\frac{1}{2}\right)^{3} \sin t, \\
\frac{\partial^{2} u}{\partial x^{2}}(1, t) & =\frac{1}{6} \sin t, \quad t>0,
\end{aligned}
$$

the exact solution in special case $\alpha=2$ is

$$
u(x, t)=\left(1+\frac{x^{5}}{120}\right) \sin t
$$

and we solve the problem (36) by variational iteration method. According to variational iteration method, formula (14) for (36) can be expressed in the following form:

$$
\begin{aligned}
& u_{n+1}(x, t) \\
& =u_{n}(x, t)-(\alpha-1) J_{t}^{\alpha} \\
& \quad \times\left(\frac{\partial^{\alpha} u_{n}(x, t)}{\partial t^{\alpha}}+\left(\frac{1}{x}+\frac{x^{4}}{120}\right) \frac{\partial^{4} u}{\partial x^{4}}\right) .
\end{aligned}
$$

Suppose that an initial approximation has the following form which satisfies the initial conditions:

$$
u_{0}(x, t)=\left(1+\frac{x^{5}}{120}\right) t
$$

Now by iteration formula (16), we obtain the following approximations:

$$
\begin{aligned}
& u_{1}(x, t) \\
& =u_{0}(x, t)-(\alpha-1) J_{t}^{\alpha} \\
& \quad \times\left(\frac{\partial^{\alpha} u_{0}(x, t)}{\partial t^{\alpha}}+\left(\frac{1}{x}+\frac{x^{4}}{120}\right) \frac{\partial^{4} u_{0}}{\partial x^{4}}\right) \\
& =\left(\left(1+\frac{x^{5}}{120}\right)\right) t-(\alpha-1)\left(1+\frac{x^{5}}{120}\right) \frac{t^{\alpha+1}}{\Gamma(\alpha+2)} .
\end{aligned}
$$

The second approximation takes the following form:

$$
\begin{aligned}
u_{2}(x, t) & \\
= & u_{1}(x, t)-(\alpha-1) J_{t}^{\alpha} \\
& \times\left(\frac{\partial^{\alpha} u_{1}(x, t)}{\partial t^{\alpha}}+\left(\frac{1}{x}+\frac{x^{4}}{120}\right) \frac{\partial^{4} u_{1}}{\partial x^{4}}\right) \\
= & \left(1+\frac{x^{5}}{120}\right) t-(\alpha-1)\left(1+\frac{x^{5}}{120}\right) \frac{t^{\alpha+1}}{\Gamma(\alpha+2)} \\
& +(\alpha-1)^{2}\left(1+\frac{x^{5}}{120}\right) \frac{t^{2 \alpha+1}}{\Gamma(2 \alpha+1)} \\
= & \left(1+\frac{x^{5}}{120}\right) \\
& \times\left(t-(\alpha-1)^{2} \frac{t^{\alpha+1}}{\Gamma(\alpha+2)}+(\alpha-1)^{2} \frac{t^{2 \alpha+1}}{\Gamma(2 \alpha+2)}\right),
\end{aligned}
$$




$$
\begin{aligned}
u_{3}(x, t) & \\
= & u_{2}(x, t)-(\alpha-1) J_{t}^{\alpha} \\
& \times\left(\frac{\partial^{\alpha} u_{2}(x, t)}{\partial t^{\alpha}}+\left(1+\frac{x^{4}}{120}\right) \frac{\partial^{4} u_{1}}{\partial x^{4}}\right) \\
= & \left(1+\frac{x^{5}}{120}\right) \\
& \times\left(t-(\alpha-1)^{3} \frac{t^{\alpha+1}}{\Gamma(\alpha+2)}+(\alpha-1)^{2}\right. \\
& \left.\quad \times \frac{t^{2 \alpha+1}}{\Gamma(2 \alpha+2)}-(\alpha-1)^{3} \frac{t^{3 \alpha+1}}{\Gamma(3 \alpha+2)}\right)
\end{aligned}
$$

Table 1 shows the absolute error of VIM solution of example (36) (when $\alpha=1.999, x=0.1$, and $n=2$ ), while Table 2 shows the maximum absolute truncated error of VIM solution (using Theorem 8) at different values of $n($ when $t=2$ ).

Example 2. Consider the following fourth-order fractional singular partial differential equation:

$$
\begin{aligned}
& \frac{\partial^{\alpha} u}{\partial t^{\alpha}}+\left(\frac{x}{\sin x}-1\right) \frac{\partial^{4} u}{\partial x^{4}}=0, \\
& 0<x<1, t>0,1<\alpha \leq 2 .
\end{aligned}
$$

With initial conditions

$$
\begin{gathered}
u(x, 0)=x-\sin x, \quad 0<x<1 \\
\frac{\partial u}{\partial t}(x, 0)=-(x-\sin x), \quad 0<x<1
\end{gathered}
$$

and boundary conditions

$$
\begin{aligned}
& u(0, t)=0, \quad u(1, t)=e^{-t}(1-\sin 1), \quad t>0, \\
& \frac{\partial^{2} u}{\partial x^{2}}(0, t)=0, \quad \frac{\partial^{2} u}{\partial x^{2}}(1, t)=e^{-t} \sin 1, \quad t>1,
\end{aligned}
$$

the exact solution in special case $\alpha=2$ is

$$
u(x, t)=(x-\sin x) e^{-t}
$$

According to variational iteration method, formula (14) for (44) can be expressed in the following form:

$$
\begin{aligned}
u_{k+1} & (x, t) \\
= & u_{k}(x, t)-(\alpha-1) J_{t}^{\alpha} \\
& \times\left(\frac{\partial^{\alpha} u_{k}(x, t)}{\partial t^{\alpha}}+\left(\frac{x}{\sin x}-1\right) \frac{\partial^{4} u}{\partial x^{4}}\right) .
\end{aligned}
$$

TABLE 1: Absolute error.

\begin{tabular}{lc}
\hline$t$ & Error of VIM $(n=2)$ \\
\hline 0.2 & $1.9635 \times 10^{-7}$ \\
0.4 & $9.44308 \times 10^{-6}$ \\
0.6 & $5.15266 \times 10^{-5}$ \\
0.8 & $1.7613 \times 10^{-4}$ \\
1 & $4.98115 \times 10^{-4}$ \\
1.2 & 0.00127331 \\
1.4 & 0.00301987 \\
1.6 & 0.00669301 \\
1.8 & 0.0139188 \\
2 & 0.02729 \\
\hline
\end{tabular}

TABLE 2: Maximum absolute error.

\begin{tabular}{lc}
\hline$n$ & Maximum error VIM \\
\hline 2 & 0.0272901 \\
3 & 0.00186871 \\
4 & 0.00328421 \\
\hline
\end{tabular}

Suppose that an initial approximation has the following form which satisfies the initial condition:

$$
u_{0}(x, t)=(x-\sin x)-(x-\sin x) t
$$

Now by iteration formula (48), we obtain the first approximation

$$
\begin{aligned}
u_{1}(x, t) & \\
= & u_{0}(x, t)-(\alpha-1) J_{t}^{\alpha} \\
& \times\left(\frac{\partial^{\alpha} u_{0}(x, t)}{\partial t^{\alpha}}+\left(\frac{x}{\sin x}-1\right) \frac{\partial^{4} u_{0}}{\partial x^{4}}\right) \\
= & (x-\sin x)-(x-\sin x) t \\
& +(\alpha-1)(x-\sin x) \frac{t^{\alpha}}{\Gamma(\alpha+1)}-(\alpha-1) \\
& \times(x-\sin x) \frac{t^{\alpha+1}}{\Gamma(\alpha+2)}
\end{aligned}
$$

and second approximation

$$
\begin{aligned}
u_{2}(x, t) & \\
= & u_{1}(x, t)-(\alpha-1) J_{t}^{\alpha} \\
& \times\left(\frac{\partial^{\alpha} u_{1}(x, t)}{\partial t^{\alpha}}+\left(\frac{x}{\sin x}-1\right) \frac{\partial^{4} u_{1}}{\partial x^{4}}\right) \\
= & (x-\sin x)-(x-\sin x) t \\
& \quad-(\alpha-3)(\alpha-1)(x-\sin x) \frac{t^{\alpha}}{\Gamma(\alpha+1)}
\end{aligned}
$$




$$
\begin{aligned}
& +(\alpha-3)(\alpha-1)(x-\sin x) \frac{t^{\alpha+1}}{\Gamma(\alpha+2)} \\
& +(\alpha-1)^{2}(x-\sin x) \frac{t^{2 \alpha}}{\Gamma(2 \alpha+1)} \\
& -(\alpha-1)^{2}(x-\sin x) \frac{t^{2 \alpha+1}}{\Gamma(2 \alpha+2)} \\
& u_{3}=(x-\sin x) \\
& \times((2-\alpha)-\alpha t \\
& +(\alpha-1)\left(\alpha^{2}-5 \alpha+7\right) \frac{t^{\alpha}}{\Gamma(\alpha+1)} \\
& +(\alpha-1)\left(\alpha^{2}-5 \alpha+5\right) \frac{t^{\alpha+1}}{\Gamma(\alpha+1)} \\
& +(\alpha-1)^{2}(2-\alpha) \frac{t^{2 \alpha}}{\Gamma(2 \alpha+1)} \\
& -(\alpha-1)^{2}(5-2 \alpha) \frac{t^{2 \alpha+1}}{\Gamma(2 \alpha+2)} \\
& \left.+(\alpha-1)^{3} \frac{t^{3 \alpha}}{\Gamma(3 \alpha+1)}-(\alpha-1)^{3} \frac{t^{3 \alpha}}{\Gamma(3 \alpha+2)}\right) \\
& u_{0}(x, t)=(x-\sin x)-(x-\sin x) t \\
& u_{1}(x, t) \\
& =u_{0}(x, t)-(\alpha-1) J_{t}^{\alpha} \\
& \times\left(\frac{\partial^{\alpha} u_{0}(x, t)}{\partial t^{\alpha}}+\left(\frac{x}{\sin x}-1\right) \frac{\partial^{4} u_{0}}{\partial x^{4}}\right) \\
& =(x-\sin x)-(x-\sin x) t \\
& +(\alpha-1)(x-\sin x) \frac{t^{\alpha}}{\Gamma(\alpha+1)} \\
& -(\alpha-1)(x-\sin x) \frac{t^{\alpha+1}}{\Gamma(\alpha+2)} \\
& =u_{1}(x, t)-(\alpha-1) J_{t}^{\alpha} \\
& \times\left(\frac{\partial^{\alpha} u_{1}(x, t)}{\partial t^{\alpha}}+\left(\frac{x}{\sin x}-1\right) \frac{\partial^{4} u_{1}}{\partial x^{4}}\right) \\
& =(x-\sin x)-(x-\sin x) t \\
& -(\alpha-3)(\alpha-1)(x-\sin x) \frac{t^{\alpha}}{\Gamma(\alpha+1)} \\
& +(\alpha-3)(\alpha-1)(x-\sin x) \frac{t^{\alpha+1}}{\Gamma(\alpha+2)}
\end{aligned}
$$$$
\begin{aligned}
& +(\alpha-1)^{2}(x-\sin x) \frac{t^{2 \alpha}}{\Gamma(2 \alpha+1)} \\
& -(\alpha-1)^{2}(x-\sin x) \frac{t^{2 \alpha+1}}{\Gamma(2 \alpha+2)}
\end{aligned}
$$$$
u_{3}(x, t)
$$$$
=(x-\sin x)
$$$$
\times((2-\alpha)-(\alpha-2) t
$$$$
+(\alpha-1)\left(\alpha^{2}-5 \alpha+7\right) \frac{t^{\alpha}}{\Gamma(\alpha+1)}
$$$$
-(\alpha-1)\left(\alpha^{2}-5 \alpha+7\right) \frac{t^{\alpha+1}}{\Gamma(\alpha+2)}
$$$$
-(\alpha-1)^{2}(2 \alpha-5) \frac{t^{2 \alpha}}{\Gamma(2 \alpha+1)}
$$$$
+(\alpha-1)^{2}(2 \alpha-5) \frac{t^{2 \alpha+1}}{\Gamma(2 \alpha+2)}
$$$$
\left.+(\alpha-1)^{3} \frac{t^{3 \alpha}}{\Gamma(3 \alpha+1)}-(\alpha-1)^{3} \frac{t^{3 \alpha+1}}{\Gamma(3 \alpha+2)}\right)
$$

Table 3 shows the absolute error of VIM solution of example (37) (when $\alpha=1.5, x=0.1$, and $n=2$ ), while Table 4 shows the maximum absolute truncated error of VIM solution (using Theorem 8) at different values of $n($ when $t=2$ ).

Example 3. Consider the following singular two-dimensional partial differential equation of fractional order:

$$
\begin{array}{r}
\frac{\partial^{\alpha} u}{\partial t^{\alpha}}+2\left(\frac{1}{x^{2}}+\frac{x^{4}}{6 !}\right) \frac{\partial^{4} u}{\partial x^{4}}+2\left(\frac{1}{y^{2}}+\frac{y^{4}}{6 !}\right) \frac{\partial^{4} u}{\partial y^{4}}=0, \\
0<x, \quad y<1, \quad t>0, \quad 1<\alpha \leq 2 .
\end{array}
$$

and boundary conditions

$$
\begin{gathered}
u(0.5, y, t)=\left(2+\frac{(0.5)^{6}}{6 !}+\frac{y^{6}}{6 !}\right) \sin t \\
u(1, y, t)=\left(2+\frac{1}{6 !}+\frac{y^{6}}{6 !}\right) \sin t, \quad t>0,
\end{gathered}
$$


TABLE 3: Absolute error.

\begin{tabular}{lc}
\hline$t$ & Error of VIM $(n=2)$ \\
\hline 0.2 & $2.9199 \times 10^{-6}$ \\
0.4 & $6.85596 \times 10^{-6}$ \\
0.6 & $7.79651 \times 10^{-6}$ \\
0.8 & $5.12596 \times 10^{-6}$ \\
1 & $1.42135 \times 10^{-6}$ \\
1.2 & $1.19864 \times 10^{-5}$ \\
1.4 & $2.66612 \times 10^{-5}$ \\
1.6 & $4.5519 \times 10^{-5}$ \\
1.8 & $6.86269 \times 10^{-5}$ \\
2 & $9.60514 \times 10^{-5}$ \\
\hline
\end{tabular}

TABLE 4: Maximum absolute error.

\begin{tabular}{lc}
\hline$n$ & Maximum error VIM \\
\hline 2 & $9.60514 \times 10^{-5}$ \\
3 & $1.82927 \times 10^{-6}$ \\
4 & $5.93438 \times 10^{-9}$ \\
\hline
\end{tabular}

$$
\begin{gathered}
\frac{\partial^{2} u}{\partial x^{2}}(0.5, y, t)=\frac{(0.5)^{4}}{6 !} \sin t, \\
\frac{\partial^{2} u}{\partial x^{2}}(1, y, t)=\frac{1}{6 !} \sin t, \quad t>1, \\
\frac{\partial^{2} u}{\partial y^{2}}(x, 0.5, t)=\frac{(0.5)^{4}}{6 !} \sin t, \\
\frac{\partial^{2} u}{\partial y^{2}}(x, 1, t)=\frac{1}{6 !} \sin t, \quad t>1,
\end{gathered}
$$

the exact solution in special case $\alpha=2$ is

$$
u(x, y, t)=\left(2+\frac{x^{6}}{6 !}+\frac{y^{6}}{6 !}\right) \sin t
$$

According to variational iteration method, formula (14) for (52) can be expressed in the following form:

$$
\begin{gathered}
u_{k+1}(x, t) \\
=u_{k}(x, t)-(\alpha-1) J_{t}^{\alpha} \\
\times\left(\frac{\partial^{\alpha} u_{k}(x, t)}{\partial t^{\alpha}}+2\left(\frac{1}{x^{2}}+\frac{x^{4}}{6 !}\right) \frac{\partial^{4} u}{\partial x^{4}}\right. \\
\left.+2\left(\frac{1}{y^{2}}+\frac{y^{4}}{6 !}\right) \frac{\partial^{4} u}{\partial y^{4}}\right) .
\end{gathered}
$$

Suppose that an initial approximation has the following form which satisfies the initial conditions:

$$
u_{0}(x, t)=\left(2+\frac{x^{6}}{6 !}+\frac{y^{6}}{6 !}\right) t .
$$

Now by iteration formula (56), we obtain the following approximations:

$$
\begin{aligned}
& u_{1}(x, t) \\
& =u_{0}(x, t)-(\alpha-1) J_{t}^{\alpha} \\
& \quad \times\left(\frac{\partial^{\alpha} u_{0}(x, t)}{\partial t^{\alpha}}+2\left(\frac{1}{x^{2}}+\frac{x^{4}}{6 !}\right) \frac{\partial^{4} u_{0}}{\partial x^{4}}\right. \\
& \left.\quad+2\left(\frac{1}{y^{2}}+\frac{y^{4}}{6 !}\right) \frac{\partial^{4} u_{0}}{\partial y^{4}}\right) \\
& =\left(2+\frac{x^{6}}{6 !}+\frac{y^{6}}{6 !}\right) t-2(\alpha-1)\left(2+\frac{x^{6}}{6 !}+\frac{y^{6}}{6 !}\right) \frac{t^{\alpha+1}}{\Gamma(\alpha+2)} .
\end{aligned}
$$

The second approximation takes the following form:

$$
\begin{aligned}
& u_{2}(x, t) \\
& =u_{1}(x, t)-(\alpha-1) J_{t}^{\alpha} \\
& \times\left(\frac{\partial^{\alpha} u_{1}(x, t)}{\partial t^{\alpha}}+2\left(\frac{1}{x^{2}}+\frac{x^{4}}{6 !}\right) \frac{\partial^{4} u_{1}}{\partial x^{4}}\right. \\
& \left.+2\left(\frac{1}{y^{2}}+\frac{y^{4}}{6 !}\right) \frac{\partial^{4} u_{1}}{\partial y^{4}}\right) \\
& =\left(2+\frac{x^{6}}{6 !}+\frac{y^{6}}{6 !}\right) t-2(\alpha-1) \\
& \times\left(2+\frac{x^{6}}{6 !}+\frac{y^{6}}{6 !}\right) \frac{t^{\alpha+1}}{\Gamma(\alpha+2)} \\
& +(\alpha-1)^{2}\left(2+\frac{x^{6}}{6 !}+\frac{y^{6}}{6 !}\right) \frac{t^{2 \alpha+1}}{\Gamma(2 \alpha+1)} \\
& =\left(2+\frac{x^{6}}{6 !}+\frac{y^{6}}{6 !}\right) \\
& \times\left(t-2(\alpha-1) \frac{t^{\alpha+1}}{\Gamma(\alpha+2)}+(\alpha-1)^{2} \frac{t^{2 \alpha+1}}{\Gamma(2 \alpha+2)}\right) \\
& u_{3}=\left(2+\frac{x^{2}}{6 !}+\frac{y^{2}}{6 !}\right) \\
& \times\left((2-\alpha) t-\alpha(2 \alpha-1) \frac{t^{\alpha+1}}{\Gamma(\alpha+2)}\right. \\
& \left.-2(\alpha-1) \frac{t^{2 \alpha+1}}{\Gamma(2 \alpha+2)}-(\alpha-1)^{3} \frac{t^{3 \alpha+1}}{\Gamma(3 \alpha+2)}\right)
\end{aligned}
$$

Table 5 shows the absolute error of VIM solution of example (38) (when $\alpha=1.999, x=y=0.1$, and $n=2$ ), while Table 6 shows the maximum absolute truncated error of VIM solution (using Theorem 8 , resp.) at different values of $n$ (when $t=2$ ). 
TABle 5: Absolute error.

\begin{tabular}{lc}
\hline$t$ & Error of VIM $(n=2)$ \\
\hline 0.2 & $4.94792 \times 10^{-6}$ \\
0.4 & $2.38092 \times 10^{-5}$ \\
0.6 & $4.09852 \times 10^{-5}$ \\
0.8 & $1.0933 \times 10^{-5}$ \\
1 & $3.29725 \times 10^{-4}$ \\
1.2 & 0.0013951 \\
1.4 & 0.00421146 \\
1.6 & 0.0106573 \\
1.8 & 0.0239528 \\
2 & 0.0492518 \\
\hline
\end{tabular}

TABLE 6: Maximum absolute error.

\begin{tabular}{lc}
\hline$n$ & Maximum error VIM \\
\hline 2 & 0.0492518 \\
3 & 0.00159092 \\
4 & 0.00124009 \\
\hline
\end{tabular}

\section{Conclusion}

The variational iteration method has been known as powerful tools for solving many equations in fractional calculus such as ordinary equations, partial differential equations, integrodifferential equations, and so many other equations. In this paper, this method has been analyzed with an aim to investigate the conditions which result in the convergence of generated series solutions of the singular partial differential equations of fractional order. The theorems outlined in the paper have proved that the approximate solutions successfully converge to the exact solution. We consider three examples to verify convergence hypothesis simplicity of the method. From the results we see that the exact error coincides with the approximate error obtained from using the theorems; for example, see Tables 1, 2, 3, and 4. Further, the high agreement of the numerical results so obtained between the variational iteration method and the exact solution in all examples reinforces the conclusion that the efficiency of this method and related phenomena give the method much wider applicability. Furthermore, the results obtained by proposed method confirm the robustness and efficiency of it. And we hope that the work in this paper is a step in this direction.

\section{Conflict of Interests}

The authors declare that there is no conflict of interests regarding the publication of this paper.

\section{Acknowledgment}

The authors express their sincere thanks to the referees for the careful and noteworthy reading of the paper, and the very helpful suggestions that improved the paper substantially.

\section{References}

[1] I. Podlubny, Fractional Differential Equations, vol. 198 of Mathematics in Science and Engineering, Academic Press, San Diego, Calif, USA, 1999.

[2] K. Diethelm and A. D. Freed, "On the solution of nonlinear fractional order differential equations used in the modeling of viscoelasticity," in Scientific Computing in Chemical Engineering II-Computational Fluid Dynamics, Reaction Engineering and Molecular Properties, F. Keil, W. Mackens, H. Voss, and J. Werther, Eds., pp. 217-224, Springer, Heidelberg, Germany, 1999.

[3] R. Metzler, W. Schick, H. Kilian, and T. F. Nonnenmacher, "Relaxation in filled polymers: A fractional calculus approach," The Journal of Chemical Physics, vol. 103, no. 16, pp. 7180-7186, 1995.

[4] R. Hilfer, Applications of Fractional Calculus in Physics, World Scientific, Singapore, 2000.

[5] K. B. Oldham and J. Spanier, The Fractional Calculus, vol. 198 of Mathematics in Science and Engineering, Academic Press, 1974.

[6] J. H. He, "Some applications of nonlinear fractional differential equations and their approximations," Bulletin of Science Technology \& Society, vol. 15, no. 2, pp. 86-90, 1999.

[7] J. H. He, "Nonlinear oscillation with fractional derivative and its applications," in Proceedings of the International Conference on Vibrating Engineering, pp. 288-291, Dalian, China, 1998.

[8] W. R. Schneider and W. Wyss, "Fractional diffusion and wave equations," Journal of Mathematical Physics, vol. 30, no. 1, pp. 134-144, 1989.

[9] S. Momani and Z. Odibat, "Numerical comparison of methods for solving linear differential equations of fractional order," Chaos, Solitons \& Fractals, vol. 31, no. 5, pp. 1248-1255, 2007.

[10] M. Abukhaled, "Variational iteration method for nonlinear singular two-point boundary value problems arising in human physiology," Journal of Mathematics, vol. 2013, Article ID 720134, 4 pages, 2013.

[11] S. Abbasbandy and E. Shivanian, "Solution of singular linear vibrational BVPs by the homotopy analysis method," Journal of Numerical Mathematics and Stochastics, vol. 1, no. 1, pp. 77-84, 2009.

[12] S. T. Mohydud-Din, A. Yildirim, M. M. Hosseini, and Y. Khan, "A study on systems of variablecoefficient singular parabolic partial differential equations," World Applied Sciences Journal, vol. 10, no. 11, pp. 1321-1327, 2010.

[13] J. He, "Variational iteration method for delay differential equations," Communications in Nonlinear Science and Numerical Simulation, vol. 2, no. 4, pp. 235-236, 1997.

[14] J. He, "Approximate solution of nonlinear differential equations with convolution product nonlinearities," Computer Methods in Applied Mechanics and Engineering, vol. 167, no. 1-2, pp. 69-73, 1998.

[15] J. He, "Approximate analytical solution for seepage flow with fractional derivatives in porous media," Computer Methods in Applied Mechanics and Engineering, vol. 167, no. 1-2, pp. 57-68, 1998.

[16] J. He, "Variational iteration method-a kind of non-linear analytical technique: Some examples," International Journal of NonLinear Mechanics, vol. 34, no. 4, pp. 699-708, 1999. 
[17] Y. Molliq R, M. S. M. Noorani, and I. Hashim, "Variational iteration method for fractional heat- and wave-like equations," Nonlinear Analysis: Real World Applications, vol. 10, no. 3, pp. 1854-1869, 2009.

[18] H. Jafari and H. Tajadodi, "He's variational iteration method for solving fractional riccati differential equation," International Journal of Differential Equations, vol. 2010, Article ID 764738, 8 pages, 2010.

[19] S. Duangpithak, "Variational iteration method for special nonlinear partial differential equations," International Journal of Mathematical Analysis, vol. 6, no. 21-24, pp. 1071-1077, 2012.

[20] F. Dal, "Application of variational iteration method to fractional hyperbolic partial differential equations," Mathematical Problems in Engineering, vol. 2009, Article ID 824385, 10 pages, 2009.

[21] S. Momani and Z. Odibat, "Comparison between the homotopy perturbation method and the variational iteration method for linear fractional partial differential equations," Computers \& Mathematics with Applications, vol. 54, no. 7-8, pp. 910-919, 2007.

[22] M. Inokuti, H. Sekine, and T. Mura, "General use of the Lagrange multiplier in non-linear mathematical physics," in Variational Method in the Mechanics of Solids, S. Nemat-Nasser, Ed., pp. 156-162, Pergamon Press, Oxford, UK, 1978.

[23] A. A. Elbeleze, A. Kılıçman, and B. M. Taib, "Note on the convergence analysis of homotopy perturbation method for fractional partial differential equations," Abstract and Applied Analysis, vol. 2014, Article ID 803902, 8 pages, 2014. 


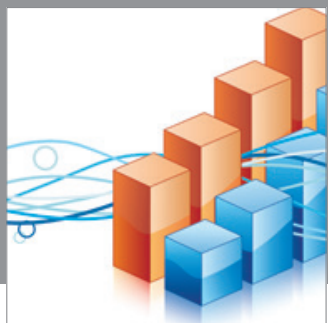

Advances in

Operations Research

mansans

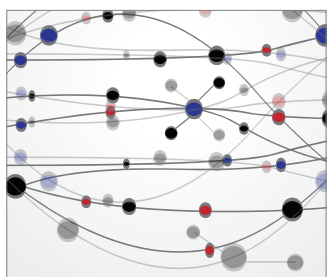

The Scientific World Journal
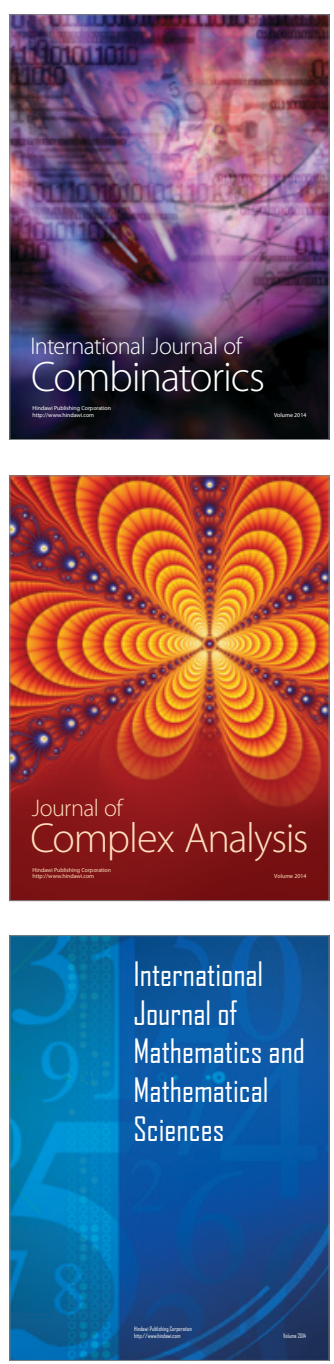
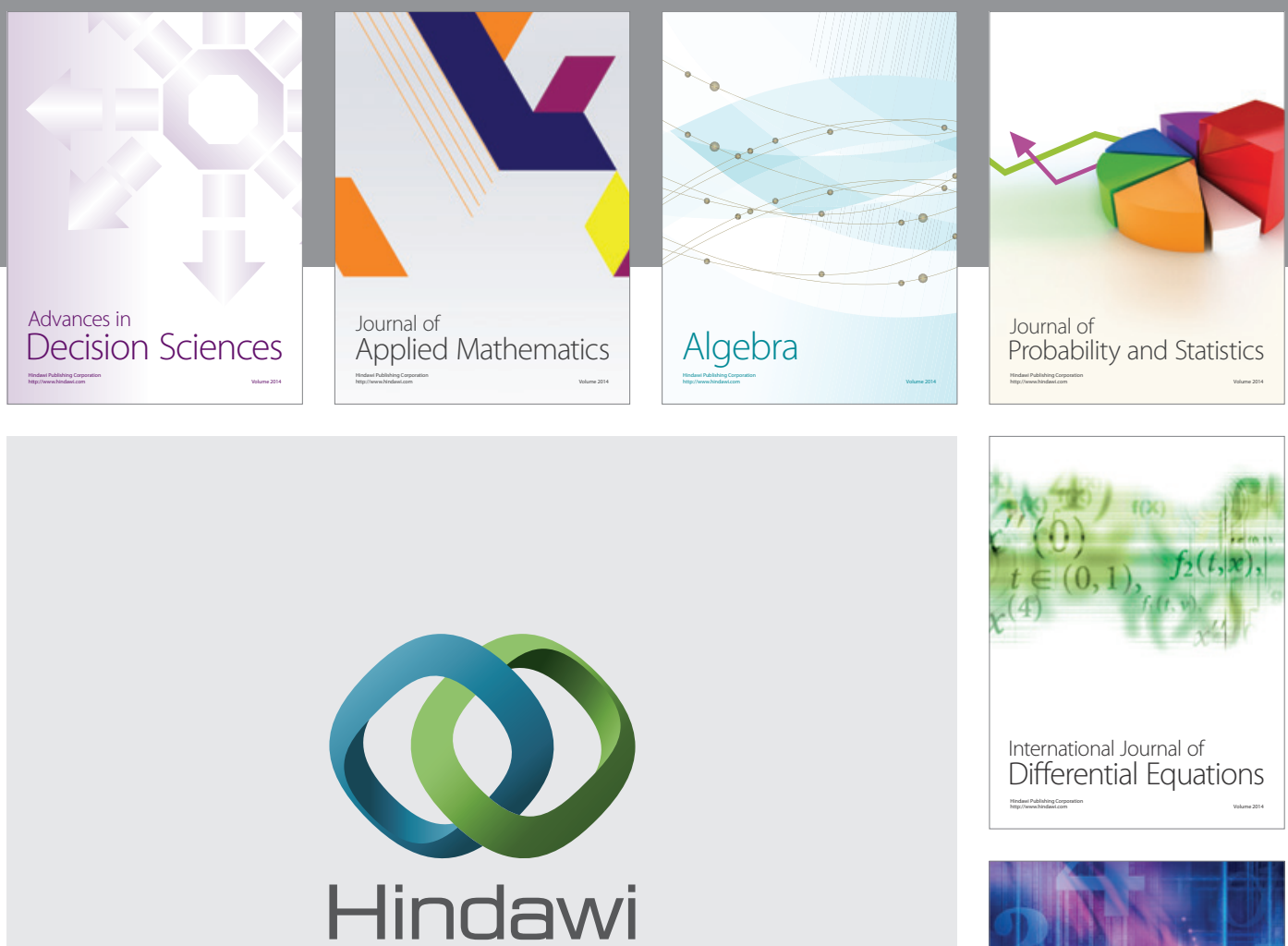

Submit your manuscripts at http://www.hindawi.com
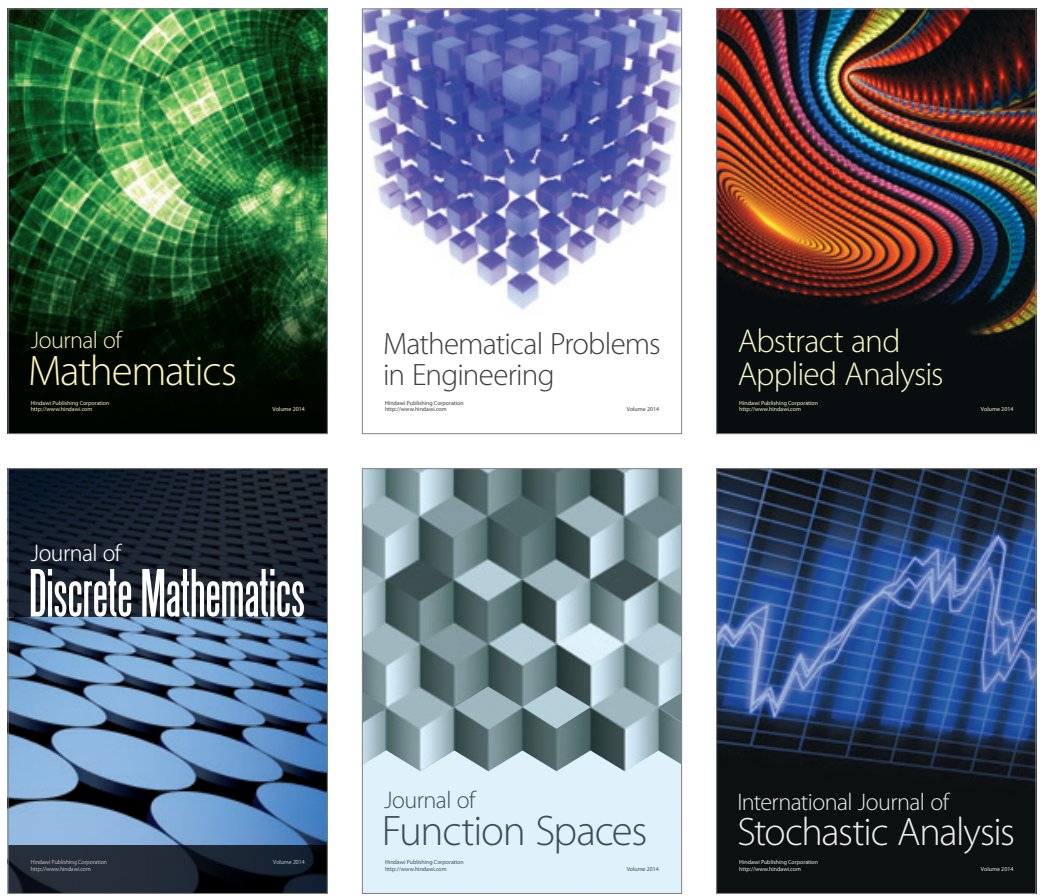

Journal of

Function Spaces

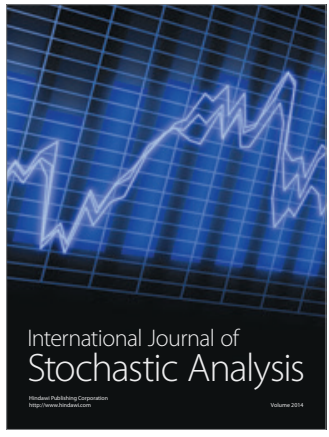

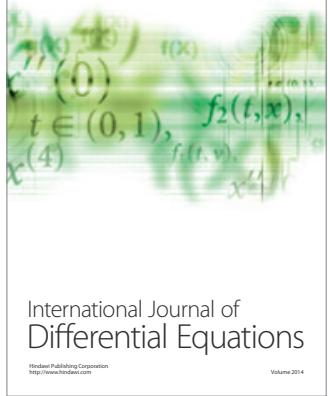
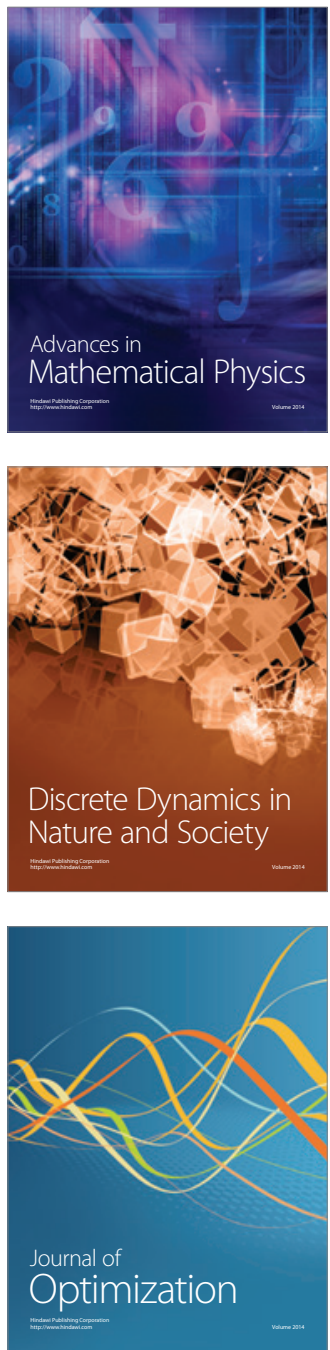